\title{
Annular Coated Inclusion model and applications for polymer nanocomposites - Part II: Cylindrical inclusions
}

\author{
Z. Wang, R.J. Oelkers, K.C. Lee, and F.T. Fisher \\ Department of Mechanical Engineering \\ Stevens Institute of Technology \\ 1 Castle Point on Hudson, Hoboken, NJ 07030 \\ Phone: 1-201-216-8913, email: Frank.Fisher@stevens.edu
}

\begin{abstract}
This work provides an analytical solution for the unknown components of the dilute strain concentration tensors for coated cylindrical inclusions, which can be directly implemented into traditional micromechanical models to predict the effective mechanical properties of composites with coated cylindrical/fibrous inclusions. Comparison of the predictions of the proposed model with predictions based on the standard Mori-Tanaka (MT) approach shows that differences between the models are largest when the annular interphase region is softer than the matrix material. A two-dimensional finite element (FE) analysis is then used to benchmark the transversely isotropic composite behavior captured in the proposed model. Using standard orientational averaging approaches, the effective moduli of composites with randomly dispersed cylindrical inclusions are predicted and compared to experimental results for polymer nanocomposites described in the literature, providing insight into the utility of the approach. In addition, by providing an analytical solution to the dilute strain concentration tensor, the proposed methodology would allow one to connect the average stress and strain fields in the constituent phases to known macroscopic fields. The proposed
\end{abstract}


model may be particularly useful as a guide to evaluate the impact various strategies that seek to tailor the properties of the interphase region in nanocomposite materials.

Keywords: Nano composites, Polymer-matrix composites (PMCs), Interphase, Modelling, Micromechanics

\section{Introduction}

The interest in polymer nanocomposites over the past years is due to their outstanding physical properties, including strength, impact consistence, and thermal stability in comparison with pure polymers [1-3]. Due to the tremendous amount of surface area available within the nanocomposite, property improvements are often attributed to the existence of a non-bulk polymer interphase in the vicinity of inclusions [4-6]. The interphase is the result of interactions between the embedded inclusions and the polymer matrix which results in local polymer properties different from that of the bulk polymer. The interphase can be a significant factor influencing the effective properties of polymer nanocomposite because of the large volume fraction of non-bulk interphase that can result from a very small nanoinclusion loading due to this surface area effect $[7,8]$.

Several micromechanical models have been developed to predict elastic properties of composites such as the well-known Mori-Tanaka method [9, 10], where the effective stiffness $C$ of a composite with a known orientational distribution of inclusions can be written as 


$$
C=\left(f^{0} C^{0}+\sum_{n=1}^{r} f^{r}\left\{C^{r} A_{d i l}^{r}\right\}\right)\left(f^{0} I+\sum_{n=1}^{r} f^{r}\left\{A_{d i l}^{r}\right\}\right)^{-1}
$$

where $f^{r}$ and $f^{\theta}$ are the volume fraction of the $r$ th inclusion phase and the matrix, respectively, brackets \{\} denote the appropriate orientational average based on the orientation distribution of the inclusions within the composite, and the dilute strain concentration tensor $A_{d i l}^{r}$ relates the average strain in the $r$ th inclusion to the average strain in the matrix such that

$$
\bar{\varepsilon}^{r}=A_{d i l}^{r} \bar{\varepsilon}^{m}
$$

For ellipsoidal inclusions, it is known that $A_{d i l}^{r}$ can be written explicitly in terms of the Eshelby tensor $S^{r}$ such that

$$
A_{d i l}^{r}=\left[I+S^{r}\left(C^{0}\right)^{-1}\left(C^{r}-C^{0}\right)\right]^{-1}
$$

where $C^{0}$ and $C^{r}$ are the stiffness tensor of the matrix and the $r$ th inclusion phase, $I$ is the identity tensor, and $S^{r}$ is the well-known Eshelby tensor. The challenge here is that the elegant solutions for the dilute strain concentration provided by the Eshelby tensor are not readily available for the physical geometry of an annular interphase region surrounding the inclusion phase. As described in [11], the Double-Inclusion model proposed by Hori and Nemat-Nasser is equivalent to the multiphase Mori-Tanaka method when the inclusion and the coating/interphase share the same aspect ratios (as is the case be studied here). A short proof is presented in the appendix of the companion paper [12]. Thus it is desirable to develop an approach that can accurately determine the dilute strain concentration tensor analytically while accounting for the true geometry of the interphase region. 
Earlier work in the literature has also examined approaches to determine the effective properties of composites with coated cylindrical inclusions. Luo and Weng evaluated the effective moduli of fiber-reinforced composite by the solutions of the average Eshelby tensor [13]. Benveniste et al. reformulated Mori-Tanaka's theory and predicted the stress field of a three-phase coated fiber composite subjected to thermoelastic loading by applying appropriate auxiliary loading cases [14]. Fisher and co-workers determined the complex moduli of a three-phase viscoelastic composite and later developed a computational finite element approach to determine the components of the dilute strain concentration factor numerically for the case of embedded wavy nanotubes $[15,16]$. To address the coated inclusion problem, Hori and Nemat-Nasser proposed a Double-inclusion model via equivalent inclusion method [11]. Using the classic self-consistent scheme, Cherkaoui et al. studied the local fields of composites containing thinly coated inclusions using Green's function and interfacial operators [17], while Seidel et al. calculated the effective properties of a carbon nanotube utilizing a composite cylinders micromechanics technique, which was then incorporated within self-consistent and Mori-Tanaka models to obtain composite effective properties [18]. An incremental damage model of particulate-reinforced composites (PRCs) based on a Mori-Tanaka mean field concept was proposed by Jiang and Tohgo et al. to study the impact of the interphase properties on the overall stress-strain response of PRCs [19].

In the companion to this work, the current authors developed an analytical solution for the components of the dilute strain concentration tensor for a coated spherical inclusion, with the result then directly available for use within the standard Mori-Tanaka approach [12]. Here, a similar approach is developed for infinitely long coated cylindrical inclusions. 
The advantage of such a formulation is that by providing an analytical solution to the components of the dilute strain concentration tensor, the proposed methodology allows one to connect the average stress and strain fields in the constituent phases to known macroscopic fields by linking the dilute strain concentration tensor to the concentration tensors using an appropriate micromechanical approach. In addition, the explicit solution to the dilute strain concentration tensor provides a straightforward path to extend the model, for example, to incorporate orientational distributions of coated inclusions (highlighted here in this paper) or to apply a spring-layer interfacial layer [20] to study the impact of imperfect interfaces on the effective response of composites with coated inclusions, which is the subject of ongoing work in our group and will be described elsewhere.

The details of the solution for the analytical derivation of the dilute strain concentration tensors for both the cylindrical inclusion and the interphase region are presented below. In addition, the predictions for the transverse composite modulus from the proposed model, a matrix-dominated property, are compared with a two-dimensional finite element (FE) model to demonstrate the validity of the proposed approach. A three-dimensional averaging method is then implemented to account for a random orientation of the coated inclusions. Lastly, predictions of the effective moduli for composites with three-dimensionally randomly dispersed coated cylindrical inclusions are compared to experimental results from the literature to demonstrate the utility of the model. 


\section{Annular Coated Inclusion (ACI) Model for Cylindrical Coated Inclusions}

\subsection{Mechanical model}

The general three-phase composite model consists of a long cylindrical inclusion, a non-bulk polymer annular interphase with thickness $t=b$ - $a$, and the bulk polymer matrix denoted by $f, g$, and $m$, respectively, as shown in Figure 1a. Using standard contracted notation, the fourth order dilute strain concentration tensors from (2) can be written explicitly for the inclusion and interphase regions as

$$
A_{d i l}^{f}=\left[\begin{array}{cccccc}
A_{11}^{f} & A_{12}^{f} & A_{13}^{f} & 0 & 0 & 0 \\
A_{21}^{f} & A_{22}^{f} & A_{23}^{f} & 0 & 0 & 0 \\
A_{31}^{f} & A_{32}^{f} & A_{33}^{f} & 0 & 0 & 0 \\
0 & 0 & 0 & A_{44}^{f} & 0 & 0 \\
0 & 0 & 0 & 0 & A_{55}^{f} & 0 \\
0 & 0 & 0 & 0 & 0 & A_{66}^{f}
\end{array}\right], A_{d i l}^{g}=\left[\begin{array}{cccccc}
A_{11}^{g} & A_{12}^{g} & A_{13}^{g} & 0 & 0 & 0 \\
A_{21}^{g} & A_{22}^{g} & A_{23}^{g} & 0 & 0 & 0 \\
A_{31}^{g} & A_{32}^{g} & A_{33}^{g} & 0 & 0 & 0 \\
0 & 0 & 0 & A_{44}^{g} & 0 & 0 \\
0 & 0 & 0 & 0 & A_{55}^{g} & 0 \\
0 & 0 & 0 & 0 & 0 & A_{66}^{g}
\end{array}\right]
$$

In the limit of the cylindrical inclusion approaching an infinite length, the dilute strain concentration tensors can be simplified into a suitable transversely isotropic form [21]. Specifically, for a cylindrical inclusion parallel to the 3 -axis with an aspect ratio $l / d \rightarrow \infty$,

$$
A_{31}^{r}=A_{32}^{r}=0, \text { and } A_{33}^{r}=1 \quad r=f, g
$$

such that the simplified dilute strain concentration tensor of each phase can be written as

$$
A_{d i l}^{f}=\left[\begin{array}{cccccc}
A_{11}^{f} & A_{12}^{f} & A_{13}^{f} & 0 & 0 & 0 \\
A_{12}^{f} & A_{11}^{f} & A_{13}^{f} & 0 & 0 & 0 \\
0 & 0 & 1 & 0 & 0 & 0 \\
0 & 0 & 0 & A_{44}^{f} & 0 & 0 \\
0 & 0 & 0 & 0 & A_{44}^{f} & 0 \\
0 & 0 & 0 & 0 & 0 & A_{66}^{f}
\end{array}\right], A_{d i l}^{g}=\left[\begin{array}{cccccc}
A_{11}^{g} & A_{12}^{g} & A_{13}^{g} & 0 & 0 & 0 \\
A_{12}^{g} & A_{11}^{g} & A_{13}^{g} & 0 & 0 & 0 \\
0 & 0 & 1 & 0 & 0 & 0 \\
0 & 0 & 0 & A_{44}^{g} & 0 & 0 \\
0 & 0 & 0 & 0 & A_{44}^{g} & 0 \\
0 & 0 & 0 & 0 & 0 & A_{66}^{g}
\end{array}\right]
$$


The number of unknown components required to determine the dilute strain concentration tensor of each phase is now reduced to five: $A_{11}^{r}, A_{12}^{r}, A_{13}^{r}, A_{44}^{r}$, and $A_{66}^{r}$. To solve these five unknown components, one can adopt the auxiliary loading cases first proposed by Benveniste et al. [14]. To briefly outline the solution procedure, one can use these auxiliary problems, which consists of a single coated inclusion embedded in a matrix, to determine the strain fields in each phase when subject to appropriate boundary conditions, from which one can determine the phase-averaged strains per unit length for the cylindrical inclusion and the annular interphase via

$$
\bar{\varepsilon}_{i j}^{r}=\frac{\int_{0}^{2 \pi} \int_{r_{i}}^{r_{o}} \varepsilon_{i j} r d r d \theta}{\pi\left[\left(r_{o}\right)^{2}-\left(r_{i}\right)^{2}\right]} .
$$

where $r_{i}$ and $r_{o}$ are the inner and outer radius of the phase. Once the average strains for each embedded phase for a particular auxiliary problem are known, one can then use the general definition of the dilute strain concentration tensor given in (2) to determine the appropriate components of $A_{d i l}^{r}$ given in (6).

Through this process, one can show that from the transverse hydrostatic loading case shown in Figure 1(b), one can obtain $A_{11}^{r}$ and $A_{12}^{r}$ as a function of $A_{66}^{r}$, which can then be determined via the transverse shear loading case shown as Figure 1 (c). The axial normal component $A_{13}^{r}$ can be obtained by superposition of the transverse hydrostatic loading case and longitudinal loading case shown as Figure 1 (d). The remaining component $A_{44}^{r}$ can be found from the longitudinal shear loading case shown in Figure 1 (e). The determination of the components of the dilute strain concentration tensor for an infinitely long, coated 
cylindrical inclusions case will be presented below. A more detailed description will be presented for the transverse hydrostatic loading case to illustrate the process; however, for brevity, only the critical steps will be outlined for the remaining auxiliary problems. Note that a similar process to analytically determine the dilute strain concentration tensor for the case of coated spherical inclusions is presented in the companion paper [12].

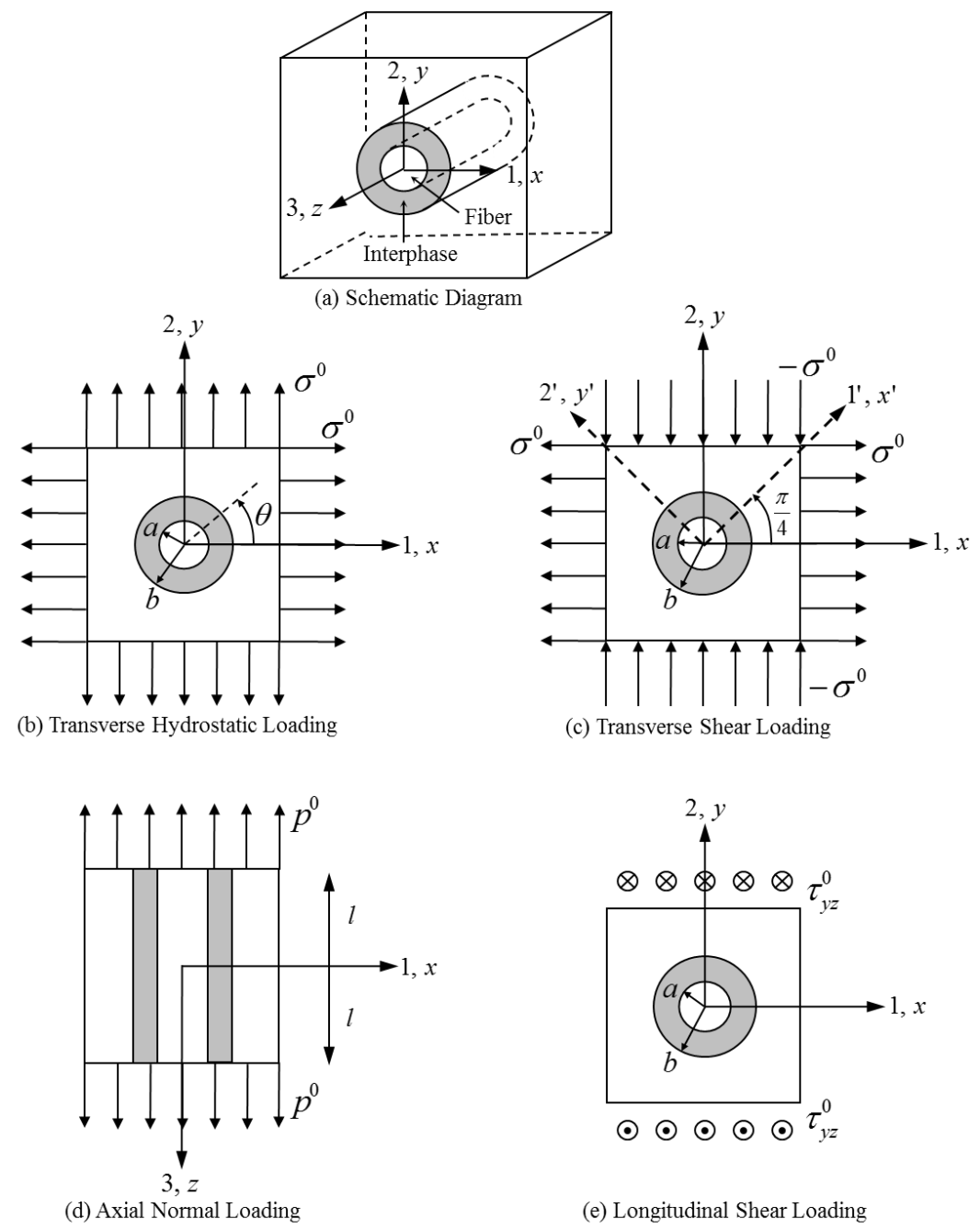

Figure 1. (a) Schematic of the coated fiber model. (b-e) Auxiliary problems used to determine the components of the dilute strain concentration tensors for the cylindrical inclusion and the annular interphase. 


\subsection{Transverse hydrostatic loading auxiliary problem}

The transverse hydrostatic loading case is shown in Figure 1 (b), where a single fiber with an annular interphase is embedded in bulk polymer matrix subject to the applied external stress $\sigma^{0}$. The loading conditions to be satisfied are

$$
\left.\sigma_{x x}\right|^{r \rightarrow \infty}=\left.\sigma_{y y}\right|^{r \rightarrow \infty}=\sigma^{0}
$$

In the limit of an infinitely long fiber one can assume a state of generalized plane-strain. The non-zero radial displacement components of the inclusion, the interphase, and the matrix can be written as

$$
u_{r}^{f}=A_{1}^{f} r \quad u_{r}^{g}=A_{1}^{g} r+\frac{B_{1}^{g}}{r^{2}} \quad u_{r}^{m}=A_{1}^{m} r+\frac{B_{1}^{m}}{r^{2}}
$$

where $A_{1}^{f}, A_{1}^{g}, B_{1}^{g}, A_{1}^{m}$, and $B_{1}^{m}$ are the unknown displacement constants, $r$ is the distance from the center of the fiber, and the subscript " 1 " denotes the transverse hydrostatic loading auxiliary problem. The radial strain components can be determined by differentiating the displacements in (9) using

$$
\varepsilon_{r r}=\frac{\partial u_{r}}{\partial r} \quad \varepsilon_{\theta \theta}=\frac{u_{r}}{r}+\frac{1}{r} \frac{\partial u_{\theta}}{\partial \theta} \quad \varepsilon_{r \theta}=\frac{1}{2}\left(\frac{1}{r} \frac{\partial u_{r}}{\partial \theta}+\frac{\partial u_{\theta}}{\partial r}-\frac{u_{\theta}}{r}\right)
$$

such that

$$
\begin{array}{ll}
\varepsilon_{r r}^{f}=A_{1}^{f} & \varepsilon_{\theta \theta}^{f}=A_{1}^{f} \\
\varepsilon_{r r}^{g}=A_{1}^{g}-\frac{B_{1}^{g}}{r^{2}} & \varepsilon_{\theta \theta}^{g}=A_{1}^{g}+\frac{B_{1}^{g}}{r^{2}} \\
\varepsilon_{r r}^{m}=A_{1}^{m}-\frac{B_{1}^{m}}{r^{2}} & \varepsilon_{\theta \theta}^{m}=A_{1}^{m}+\frac{B_{1}^{m}}{r^{2}}
\end{array}
$$


As $r \rightarrow \infty$, the far-field strains can be written as

$$
\left.\varepsilon_{r r}^{m}\right|^{r \rightarrow \infty}=A_{1}^{m}=\left.\varepsilon_{r r}^{0} \quad \varepsilon_{\theta \theta}^{m}\right|^{r \rightarrow \infty}=A_{1}^{m}=\varepsilon_{\theta \theta}^{0}
$$

The non-zero stress components can be found by applying Hooke's Law

$$
\sigma_{i j}=2 \mu \varepsilon_{i j}+\lambda \varepsilon_{k k} \delta_{i j}
$$

where $\lambda$ and $\mu$ are the first and second Lamé constants and $\delta_{i j}$ is the Kronecker delta. The non-zero stress components can then be written as

$$
\begin{array}{ll}
\sigma_{r r}^{f}=2 A_{1}^{f}\left(\mu_{y z}^{f}+\lambda_{y z}^{f}\right) & \sigma_{\theta \theta}^{f}=2 A_{1}^{f}\left(\mu_{y z}^{f}+\lambda_{y z}^{f}\right) \\
\sigma_{r r}^{g}=2 A_{1}^{g}\left(\mu_{y z}^{g}+\lambda_{y z}^{g}\right)-2 \mu_{y z}^{g} \frac{B_{1}^{g}}{r^{2}} & \sigma_{\theta \theta}^{g}=2 A_{1}^{g}\left(\mu_{y z}^{g}+\lambda_{y z}^{g}\right)+2 \mu_{y z}^{g} \frac{B_{1}^{g}}{r^{2}} \\
\sigma_{r r}^{m}=2 A_{1}^{m}\left(\mu_{y z}^{m}+\lambda_{y z}^{m}\right)-2 \mu_{y z}^{m} \frac{B_{1}^{m}}{r^{2}} & \sigma_{\theta \theta}^{m}=2 A_{1}^{m}\left(\mu_{y z}^{m}+\lambda_{y z}^{m}\right)+2 \mu_{y z}^{m} \frac{B_{1}^{m}}{r^{2}}
\end{array}
$$

Similarly, the far-field stress can be determined as $r \rightarrow \infty$ such that

$$
\begin{aligned}
& \left.\sigma_{r r}^{m}\right|^{r \rightarrow \infty}=2 A_{1}^{m}\left(\mu_{y z}^{m}+\lambda_{y z}^{m}\right)=\sigma_{r r}^{0} \\
& \left.\sigma_{\theta \theta}^{m}\right|^{r \rightarrow \infty}=2 A_{1}^{m}\left(\mu_{y z}^{m}+\lambda_{y z}^{m}\right)=\sigma_{\theta \theta}^{0}
\end{aligned}
$$

Assuming perfect bonding between the phases, the appropriate boundary conditions for the transverse hydrostatic auxiliary problem are

$$
\begin{array}{ll}
u_{r}^{f}=u_{r}^{g}, \sigma_{r r}^{f}=\sigma_{r r}^{g} & \text { at } r=a \\
u_{r}^{g}=u_{r}^{m}, \sigma_{r r}^{g}=\sigma_{r r}^{m} & \text { at } r=b \\
\sigma_{r r}^{m}=\sigma^{0} & \text { as } r \rightarrow \infty
\end{array}
$$

Substituting (9), (14) and (15) into the boundary conditions (16), the original five unknown displacement constants $A_{1}^{f}, A_{1}^{g}, B_{1}^{g}, A_{1}^{m}$, and $B_{1}^{m}$ from (9) can be determined via solution of 
the following system of equations

$$
\left[\begin{array}{c}
A_{1}^{f} \\
A_{1}^{g} \\
B_{1}^{g} \\
A_{1}^{m} \\
B_{1}^{m}
\end{array}\right]=\left[\begin{array}{ccccc}
a & -a & -\frac{1}{a} & 0 & 0 \\
0 & b & \frac{1}{b} & -b & -\frac{1}{b} \\
\left(\mu_{y z}^{f}+\lambda_{y z}^{m}\right) & -\left(\mu_{y z}^{g}+\lambda_{y z}^{m}\right) & \frac{\mu_{y z}^{g}}{a^{2}} & 0 & 0 \\
0 & \left(\mu_{y z}^{g}+\lambda_{y z}^{m}\right) & -\frac{\mu_{y z}^{g}}{b^{2}} & -\left(\mu_{y z}^{m}+\lambda_{y z}^{m}\right) & \frac{\mu_{y z}^{m}}{b^{2}} \\
0 & 0 & 0 & 2\left(\mu_{y z}^{m}+\lambda_{y z}^{m}\right) & 0
\end{array}\right]^{-1} \cdot\left[\begin{array}{c}
0 \\
0 \\
0 \\
0 \\
\sigma^{0}
\end{array}\right]
$$

After solving for the unknown constants in the displacement equations given in (9), the radial strain and stress components can be easily determined from Error! Reference source not found.) and (14), respectively.After transforming the strains to the Cartesian coordinate system prior to integration to simplify the analysis, the average strain in the inclusion and annular interphase region can then be determined from (7) such that

$$
\begin{array}{lll}
\bar{\varepsilon}_{x x}^{f}=A_{1}^{f} & \bar{\varepsilon}_{y y}^{f}=A_{1}^{f} & \bar{\varepsilon}_{x y}^{f}=0 \\
\bar{\varepsilon}_{x x}^{g}=A_{1}^{g} & \bar{\varepsilon}_{y y}^{g}=A_{1}^{g} & \bar{\varepsilon}_{x y}^{g}=0
\end{array}
$$

where as $r \rightarrow \infty$ the far-field strain in the matrix can be written as

$$
\bar{\varepsilon}_{x x}^{0}=\bar{\varepsilon}_{y y}^{0}=A_{1}^{m}
$$

Using the definition of the dilute strain concentration tensor given in (2) and the assumption of generalized plane strain for this auxiliary problem, the average strain in the inclusion and interphase can be written as

$$
\begin{array}{ll}
\bar{\varepsilon}_{x x}^{f}=A_{11}^{f} \bar{\varepsilon}_{x x}^{0}+A_{12}^{f} \bar{\varepsilon}_{y y}^{0} & \bar{\varepsilon}_{y y}^{f}=A_{12}^{f} \bar{\varepsilon}_{x x}^{0}+A_{11}^{f} \bar{\varepsilon}_{y y}^{0} \\
\bar{\varepsilon}_{x x}^{g}=A_{11}^{g} \bar{\varepsilon}_{x x}^{0}+A_{12}^{g} \bar{\varepsilon}_{y y}^{0} & \bar{\varepsilon}_{y y}^{g}=A_{12}^{g} \bar{\varepsilon}_{x x}^{0}+A_{11}^{g} \bar{\varepsilon}_{y y}^{0}
\end{array}
$$

From (18) and 
(19), the sum of factors $A_{11}^{r}$ and $A_{12}^{r}$ can then be determined as

$$
A_{11}^{r}+A_{12}^{r}=\frac{\bar{\varepsilon}_{x x}^{f}}{\bar{\varepsilon}_{x x}^{0}}=\frac{A_{1}^{r}}{A_{1}^{m}} \quad(r=f, g)
$$

Given transverse isotropy of the system, the shear component $A_{66}^{r}$ can be written as

$$
A_{66}^{r}=A_{11}^{r}-A_{12}^{r}
$$

By combining (21) and (22), $A_{11}^{r}$ and $A_{12}^{r}$ can be written as

$$
\begin{aligned}
& A_{11}^{r}=A_{22}^{r}=\frac{1}{2}\left(\frac{A_{1}^{r}}{A_{1}^{m}}+A_{66}^{r}\right) \\
& A_{12}^{r}=A_{21}^{r}=\frac{1}{2}\left(\frac{A_{1}^{r}}{A_{1}^{m}}-A_{66}^{r}\right)
\end{aligned}
$$

where $A_{66}^{r}$ can be determined via the transverse shear auxiliary problem presented below.

\subsection{Transverse shear loading auxiliary problem}

As shown in Figure 1(c), the transverse shear loading case is described by the loading conditions

$$
\left.\sigma_{x x}\right|^{r \rightarrow \infty}=-\left.\sigma_{y y}\right|^{r \rightarrow \infty}=\sigma^{0}
$$

where again a state of generalized plane-strain is assumed. Based on the work of Christensen et al. [22] and Benveniste et al. [14], the radial displacement components for the transverse shear loading case are 


$$
\begin{aligned}
& \left.u_{r}^{f}=\left(\frac{b}{4 \mu_{x y}^{f}}\right)\left[A_{2}^{f}\left(\eta^{f}-3\right)\left(\frac{r}{b}\right)^{3}+D_{2}^{f}\left(\frac{r}{b}\right)\right] \cos 2 \theta\right] \\
& u_{\theta}^{f}=\left(\frac{b}{4 \mu_{x y}^{f}}\right)\left[A_{2}^{f}\left(\eta^{f}+3\right)\left(\frac{r}{b}\right)^{3}-D_{2}^{f}\left(\frac{r}{b}\right)\right] \sin 2 \theta \leq a \\
& \left.u_{r}^{g}=\left(\frac{b}{4 \mu_{x y}^{g}}\right)\left[A_{2}^{g}\left(\eta^{g}-3\right)\left(\frac{r}{b}\right)^{3}+D_{2}^{g}\left(\frac{r}{b}\right)+C_{2}^{g}\left(\eta^{g}+1\right)\left(\frac{b}{r}\right)+B_{2}^{g}\left(\frac{b}{r}\right)^{3}\right] \cos 2 \theta\right] \\
& \left.u_{\theta}^{g}=\left(\frac{b}{4 \mu_{x y}^{g}}\right)\left[A_{2}^{g}\left(\eta^{g}+3\right)\left(\frac{r}{b}\right)^{3}-D_{2}^{g}\left(\frac{r}{b}\right)-C_{2}^{g}\left(\eta^{g}-1\right)\left(\frac{b}{r}\right)+B_{2}^{g}\left(\frac{b}{r}\right)^{3}\right] \sin 2 \theta\right] \\
& \left.u_{r}^{m}=\left(\frac{b}{4 \mu_{x y}^{m}}\right)\left[2\left(\frac{r}{b}\right)+C_{2}^{m}\left(\eta^{m}+1\right)\left(\frac{b}{r}\right)+B_{2}^{m}\left(\frac{b}{r}\right)^{3}\right] \cos 2 \theta\right] \\
& \left.u_{\theta}^{m}=\left(\frac{b}{4 \mu_{x y}^{m}}\right)\left[-2\left(\frac{r}{b}\right)-C_{2}^{m}\left(\eta^{m}-1\right)\left(\frac{b}{r}\right)+B_{2}^{m}\left(\frac{b}{r}\right)^{3}\right] \sin 2 \theta\right\} r<\infty
\end{aligned}
$$

where $A_{2}^{f}, A_{2}^{g}, B_{2}^{g}, B_{2}^{m}, C_{2}^{g}, C_{2}^{m}, D_{2}^{f}, D_{2}^{g}$ are the unknown displacement constants, the subscript " 2 " denotes the transverse shear auxiliary problem, and the term $\eta^{r}$ is the Muskhelishvili coefficient [23] defined as

$$
\eta^{r}=1+2 \frac{\mu_{x y}^{r}}{K^{r}}
$$

where $\mu_{x y}^{r}$ and $K^{r}$ are the transverse shear and the plane strain bulk modulus of each phase. Given these displacements, the strain and stress components of each phase can be readily determined using the procedure outlined in the previous section. The far-field strain can be obtained as $r \rightarrow \infty$ such that 


$$
\begin{aligned}
& \varepsilon_{r r}^{0}=\left.\varepsilon_{r r}^{m}\right|^{r \rightarrow \infty}=\left(\frac{1}{2 \mu_{x y}^{m}}\right) \cos (2 \theta) \\
& \varepsilon_{\theta \theta}^{0}=\left.\varepsilon_{\theta \theta}^{m}\right|^{r \rightarrow \infty}=-\left(\frac{1}{2 \mu_{x y}^{m}}\right) \cos (2 \theta) \\
& \varepsilon_{r \theta}^{0}=\left.\varepsilon_{r \theta}^{m}\right|^{r \rightarrow \infty}=-\left(\frac{1}{2 \mu_{x y}^{m}}\right) \sin (2 \theta)
\end{aligned}
$$

The unknown displacement constants for this auxiliary problem can be determined by satisfying the applicable boundary conditions

$$
\begin{aligned}
& \left.\begin{array}{l}
u_{r}^{f}=u_{r}^{g}, u_{\theta}^{f}=u_{\theta}^{g} \\
\sigma_{r r}^{f}=\sigma_{r r}^{g}, \sigma_{r \theta}^{f}=\sigma_{r \theta}^{g}
\end{array}\right\} \quad \text { at } r=a \\
& \left.\begin{array}{l}
u_{r}^{g}=u_{r}^{m}, u_{\theta}^{g}=u_{\theta}^{m} \\
\sigma_{r r}^{g}=\sigma_{r r}^{m}, \sigma_{r \theta}^{g}=\sigma_{r \theta}^{m}
\end{array}\right\} \quad \text { at } r=b
\end{aligned}
$$

These eight unknowns $A_{2}^{f}, A_{2}^{g}, C_{2}^{m}, B_{2}^{g}, C_{2}^{g}, B_{2}^{m}, D_{2}^{f}, D_{2}^{g}$ be determined via the solution of the system of equations given in the Appendix.

With the eight constants in the displacement equations found, the strain and stress fields in each phase are explicitly known, where transformation to the $\left(x^{\prime}, y^{\prime}\right)$ Cartesian coordinate system in Figure 1(c) will simplify the analysis. After volume averaging, the non-zero shear strain components of the inclusion, interphase, and at the far-field can be written as

$$
\begin{aligned}
& \bar{\varepsilon}_{x^{\prime} y^{\prime}}^{f}=\left(\frac{1}{4 \mu_{x y}^{f}}\right)\left[D_{2}^{f}-3 A_{2}^{f}\left(\frac{a}{b}\right)^{2}\right] \\
& \bar{\varepsilon}_{x^{\prime} y^{\prime}}^{g}=\left(\frac{1}{4 \mu_{x y}^{g}}\right)\left[D_{2}^{g}-3 A_{2}^{g}\left(\frac{a^{2}+b^{2}}{b^{2}}\right)\right] \\
& \varepsilon_{x^{\prime} y^{\prime}}^{\infty}=\frac{1}{2 \mu_{x y}^{m}}
\end{aligned}
$$


From the definition of the dilute strain concentration tensor in (2), the transverse shear component of the dilute strain concentration tensor $A_{66}^{r}$ for the inclusion and interphase can be written as

$$
\begin{aligned}
& A_{66}^{f}=\frac{\bar{\varepsilon}_{x^{\prime} y^{\prime}}^{f}}{\varepsilon_{x^{\prime} y^{\prime}}^{0}}=\frac{\mu_{x y}^{m}}{2 \mu_{x y}^{f}}\left[D_{2}^{f}-3 A_{2}^{f}\left(\frac{a}{b}\right)^{2}\right] \\
& A_{66}^{g}=\frac{\bar{\varepsilon}_{x^{\prime} y^{\prime}}^{g}}{\varepsilon_{x^{\prime} y^{\prime}}^{0}}=\frac{\mu_{x y}^{m}}{2 \mu_{x y}^{g}}\left[D_{2}^{g}-3 A_{2}^{g}\left(\frac{a^{2}+b^{2}}{b^{2}}\right)\right]
\end{aligned}
$$

One can now substitute $A_{66}^{r}$ into (23) to find the values for $A_{11}^{r}=A_{22}^{r}$ and $A_{12}^{r}=A_{21}^{r}$.

\subsection{Axial normal loading auxiliary problem}

The axial strain concentration factors $A_{13}^{r}$ can be determined via superposition of Figure 1(b) and Figure 1(d). Here the stress and strain fields about the z-axis must be considered; reformulating the first auxiliary problem as suggested by Benveniste et al. is a convenient way to solve this loading case [14]. The external loading conditions that must be satisfied for the axial normal loading case are

$$
\begin{aligned}
& \left.\sigma_{x x}\right|^{r \rightarrow \infty}=\sigma_{x x}^{0} \\
& \left.\sigma_{y y}\right|^{r \rightarrow \infty}=\sigma_{y y}^{0} \\
& \left.\sigma_{z z}\right|^{z \rightarrow \pm L}=p^{0}
\end{aligned}
$$

The radial displacement components can be written as [14] 


$$
\begin{aligned}
& u_{r}^{f}=A_{3}^{f} r \\
& u_{r}^{g}=A_{3}^{g} r+\frac{B_{3}^{g}}{r} \\
& u_{r}^{m}=A_{3}^{m} r+\frac{B_{3}^{m}}{r}
\end{aligned}
$$

Strain and stress components can be derived by appropriate differentiation of the displacement equations following the procedure illustrated previously. Note that the external load $p^{0}$ is applied on all three phases, so the far-field stress and strain about the $\mathrm{z}$ axis can be written as

$$
\begin{array}{cc}
\left.\sigma_{z z}^{r}\right|^{z \rightarrow \pm L}=p^{0} & r=f, g, m \\
\left.\varepsilon_{z z}^{r}\right|^{z \rightarrow \pm L}=\varepsilon_{z z}^{0}=\frac{p^{0}}{\bar{E}_{z z}} & r=f, g, m
\end{array}
$$

where the effective composite modulus in the axial direction $\bar{E}_{z z}=f^{f} E_{z z}^{f}+f^{g} E_{z z}^{g}+f^{m} E_{z z}^{m}$ can be obtained from the standard Rule of Mixtures. Continuity of $u_{r}, \sigma_{r r}$, and $\sigma_{z z}$ requires that

$$
\begin{array}{rlrl}
u_{r}^{f}=u_{r}^{g}, & \sigma_{r r}^{f}=\sigma_{r r}^{g} & \text { at } r=a \\
u_{r}^{g}=u_{r}^{m}, & \sigma_{r r}^{g}=\sigma_{r r}^{m} & \text { at } r=b \\
\sigma_{z z}^{r}=p^{0} & \text { at } z= \pm L
\end{array}
$$

From these boundary conditions, the five unknown constants $A_{3}^{f}, A_{3}^{g}, B_{3}^{g}, A_{3}^{m}$ and $B_{3}^{m}$ in (32) can be determined by solving the following system of equations 


$$
\left[\begin{array}{l}
A_{3}^{f} \\
A_{3}^{g} \\
B_{3}^{g} \\
A_{3}^{m} \\
B_{3}^{m}
\end{array}\right]=\left[\begin{array}{ccccc}
a & -a & -\frac{1}{a} & 0 & 0 \\
0 & b & \frac{1}{b} & -b & -\frac{1}{b} \\
2\left(\mu_{y z}^{f}+\lambda^{f}\right) & -2\left(\mu_{y z}^{g}+\lambda^{g}\right) & 2 \frac{\mu_{y z}^{g}}{a^{2}} & 0 & 0 \\
0 & 2\left(\mu_{y z}^{g}+\lambda^{g}\right) & -2 \frac{\mu_{y z}^{g}}{b^{2}} & -2\left(\mu_{y z}^{m}+\lambda^{m}\right) & 2 \frac{\mu_{y z}^{m}}{b^{2}} \\
2 \lambda^{f} f^{f} & 2 \lambda^{g} f^{g} & 0 & 2 \lambda^{m} f^{m} & 0
\end{array}\right]^{-1} \cdot\left[\begin{array}{c}
0 \\
0 \\
\varepsilon_{z z}^{0}\left(\lambda^{g}-\lambda^{f}\right) \\
\varepsilon_{z z}^{0}\left(\lambda^{m}-\lambda^{g}\right) \\
\xi
\end{array}\right]
$$

where

$$
\xi=p^{0}-2 \varepsilon_{z z}^{0}\left(\lambda^{m} v_{y z}^{m} f^{m}+\lambda^{g} v_{y z}^{g} f^{g}+\lambda^{f} v_{y z}^{f} f^{f}\right)
$$

With the five unknown constants in the displacement equations, the radial strain and stress components can now be determined. After appropriate volume averaging and conversion to Cartesian coordinates, the non-zero average strains in the phases are found to be

$$
\begin{array}{lll}
\bar{\varepsilon}_{x x}^{f}=A_{3}^{f} & \bar{\varepsilon}_{y y}^{f}=A_{3}^{f} & \bar{\varepsilon}_{z z}^{f}=\frac{p^{0} f^{f}}{E_{z z}^{f} f^{f}+E_{z z}^{g} f^{g}+E_{z z}^{m} f^{m}} \\
\bar{\varepsilon}_{x x}^{g}=A_{3}^{g} & \bar{\varepsilon}_{y y}^{g}=A_{3}^{g} & \bar{\varepsilon}_{z z}^{g}=\frac{p^{0} f^{g}}{E_{z z}^{f} f^{f}+E_{z z}^{g} f^{g}+E_{z z}^{m} f^{m}} \\
\bar{\varepsilon}_{x x}^{m}=A_{3}^{m} & \bar{\varepsilon}_{y y}^{m}=A_{3}^{m} & \bar{\varepsilon}_{z z}^{m}=\frac{p^{0} f^{m}}{E_{z z}^{f} f^{f}+E_{z z}^{g} f^{g}+E_{z z}^{m} f^{m}}
\end{array}
$$

From the definition of the dilute strain concentration tensor (2), the average strains in the cylindrical fiber and annular interphase can be written as

$$
\begin{array}{ll}
\bar{\varepsilon}_{x x}^{f}=A_{11}^{f} \bar{\varepsilon}_{x x}^{0}+A_{12}^{f} \bar{\varepsilon}_{y y}^{0}+A_{13}^{f} \bar{\varepsilon}_{z z}^{0} & \bar{\varepsilon}_{y y}^{f}=A_{21}^{f} \bar{\varepsilon}_{x x}^{0}+A_{22}^{f} \bar{\varepsilon}_{y y}^{0}+A_{23}^{f} \bar{\varepsilon}_{z z}^{0} \\
\bar{\varepsilon}_{x x}^{g}=A_{11}^{g} \overline{\bar{c}}_{x x}^{0}+A_{12}^{g} \bar{\varepsilon}_{y y}^{0}+A_{13}^{g} \bar{\varepsilon}_{z z}^{0} & \bar{\varepsilon}_{y y}^{g}=A_{21}^{g} \bar{\varepsilon}_{x x}^{0}+A_{22}^{g} \bar{\varepsilon}_{y y}^{0}+A_{23}^{g} \bar{\varepsilon}_{z z}^{0}
\end{array}
$$

From (38) and (39), the dilute strain concentration factors can be determined as 


$$
\begin{aligned}
& A_{13}^{f}=\frac{\bar{\varepsilon}_{x x}^{g}-\left(A_{11}^{f} \bar{\varepsilon}_{x x}^{0}+A_{12}^{f} \bar{\varepsilon}_{y y}^{0}\right)}{\bar{\varepsilon}_{z z}^{0}}=\frac{A_{3}^{f}-A_{3}^{m}\left(A_{11}^{f}+A_{12}^{f}\right)}{\bar{\varepsilon}_{z z}^{0}} \\
& A_{13}^{g}=\frac{\bar{\varepsilon}_{x x}^{g}-\left(A_{21}^{g} \bar{\varepsilon}_{x x}^{0}+A_{22}^{g} \bar{\varepsilon}_{y y}^{0}\right)}{\bar{\varepsilon}_{z z}^{0}}=\frac{A_{3}^{g}-A_{3}^{m}\left(A_{11}^{g}+A_{12}^{g}\right)}{\bar{\varepsilon}_{z z}^{0}}
\end{aligned}
$$

where $A_{11}^{r}$ and $A_{12}^{r}$ were determined previously via (23) and $A_{22}^{r}=A_{11}^{r}$ and $A_{21}^{r}=A_{12}^{r}$ due to the transverse isotropy of the dilute strain concentration tensor (6).

\subsection{Axial shear auxiliary problem}

The axial shear loading case is shown as Figure 1(e), where the appropriate boundary condition is

$$
\left.\tau_{y z}^{m}\right|_{r \rightarrow \infty}=\tau_{y z}^{0}
$$

Here the non-zero displacement equations can be written as [14]

$$
u_{z}^{f}=A_{4}^{f} r \sin \theta \quad u_{z}^{g}=\left(A_{4}^{g} r+\frac{B_{4}^{g}}{r}\right) \sin \theta \quad u_{z}^{m}=\left(A_{4}^{m} r+\frac{B_{4}^{m}}{r}\right) \sin \theta
$$

Necessary boundary conditions that must be satisfied for this auxiliary problem are

$$
\begin{aligned}
& u_{z}^{f}=u_{z}^{g}, \quad \sigma_{r z}^{f}=\sigma_{r z}^{g} \quad \text { at } r=a \\
& u_{z}^{g}=u_{z}^{m}, \quad \sigma_{r z}^{g}=\sigma_{r z}^{m} \quad \text { at } r=b \\
& \sigma_{r z}^{m}=\sigma_{r z}^{0} \quad \text { as } r \rightarrow \infty
\end{aligned}
$$

Based on these boundary conditions, one can show that the five unknown displacement equation constants $A_{4}^{f}, A_{4}^{g}, B_{4}^{g}, A_{4}^{m}$, and $B_{4}^{m}$ can be determined, after conversion to Cartesian coordinates, based on the solution to the following system of equations 


$$
\left[\begin{array}{l}
A_{4}^{f} \\
A_{4}^{g} \\
B_{4}^{g} \\
A_{4}^{m} \\
B_{4}^{m}
\end{array}\right]=\left[\begin{array}{ccccc}
a & -a & -\frac{1}{a} & 0 & 0 \\
0 & b & \frac{1}{b} & -b & -\frac{1}{b} \\
\mu_{y z}^{f} & -\mu_{y z}^{g} & \frac{\mu_{y z}^{g}}{a^{2}} & 0 & 0 \\
0 & \mu_{y z}^{g} & -\frac{\mu_{y z}^{g}}{a^{2}} & -\mu_{y z}^{m} & \frac{\mu_{y z}^{m}}{b^{2}} \\
0 & 0 & 0 & \mu_{y z}^{m} & 0
\end{array}\right]^{-1} \cdot\left[\begin{array}{c}
0 \\
0 \\
0 \\
0 \\
\tau_{y z}^{0}
\end{array}\right]
$$

Using (7), the average strain terms for each phase are then found to be

$$
\bar{\varepsilon}_{y z}^{f}=\frac{1}{2} A_{4}^{f} \quad \bar{\varepsilon}_{y z}^{g}=\frac{1}{2} A_{4}^{g} \quad \bar{\varepsilon}_{y z}^{m}=\frac{1}{2} A_{4}^{m}
$$

From (2), the unknown components of the dilute strain concentration tensor $A_{44}^{r}$ and $A_{55}^{r}$ can now be determined as

$$
A_{44}^{f}=A_{55}^{f}=\frac{A_{4}^{f}}{A_{4}^{m}} \quad A_{44}^{g}=A_{55}^{g}=\frac{A_{4}^{g}}{A_{4}^{m}}
$$

\subsection{Implementation within the Mori-Tanaka method for orientational distributions of coated cylindrical inclusions}

Based on the solution of the auxiliary problems above, the dilute strain concentration tensor for the fiber and the annular interphase region can be directly used in a standard micromechanics approach. Note that, as one would expect, when the inclusion and the interphase are the same material, the solution provided with the proposed approach is identical to that provided by an equivalent two phase model.

A particular utility of the analytical determination of the components of the dilute strain concentration tensors is that it can be readily adapted to consider cases where the inclusions 
are dispersed in a matrix with a known orientational distribution, for example using the Mori-Tanaka approach described earlier. Here the orientational averages of the terms $\left\{C^{r} A_{d i l}^{r}\right\}$ and $\left\{A_{d i}^{r}\right\}$ in (1) can be obtained using spectral decomposition [24, 25].

As an example, consider the case where one wishes to determine the 3D random orientational average of a fourth-order tensor expressed in contracted notation $L_{i j}$. The corresponding orientational average of $\left\{L_{i j}\right\}$ can be expressed as

$$
\left\{L_{i j}\right\}=\alpha J_{i j}+\beta K_{i j}
$$

where for a $3 \mathrm{D}$ random orientation the scalars $\alpha$ and $\beta$ can be written as

$$
\alpha=\frac{1}{3} \sum_{j=1}^{3} \sum_{i=1}^{3} L_{i j} \quad \beta=\frac{1}{5} \sum_{j=1}^{6} \sum_{i=1}^{6} L_{i j} \delta_{i j}-\frac{1}{15} \sum_{j=1}^{3} \sum_{i=1}^{3} L_{i j}
$$

while the tensors $J_{i j}$ and $K_{i j}$ in (48) can be written in contracted notation as

$$
J_{i j}=\frac{1}{3}\left[\begin{array}{llllll}
1 & 1 & 1 & 0 & 0 & 0 \\
1 & 1 & 1 & 0 & 0 & 0 \\
1 & 1 & 1 & 0 & 0 & 0 \\
0 & 0 & 0 & 0 & 0 & 0 \\
0 & 0 & 0 & 0 & 0 & 0 \\
0 & 0 & 0 & 0 & 0 & 0
\end{array}\right], \quad K_{i j}=\frac{1}{3}\left[\begin{array}{cccccc}
2 & -1 & -1 & 0 & 0 & 0 \\
-1 & 2 & -1 & 0 & 0 & 0 \\
-1 & -1 & 2 & 0 & 0 & 0 \\
0 & 0 & 0 & 3 & 0 & 0 \\
0 & 0 & 0 & 0 & 3 & 0 \\
0 & 0 & 0 & 0 & 0 & 3
\end{array}\right]
$$

In this manner, the 3D random orientational average of a fourth order tensor can be readily found. 


\section{Finite Element Analysis}

To examine the predictions of the original Mori-Tanaka (MT) method, where the dilute strain concentration tensor for each phase is approximated using the standard Eshelby tensor, and the proposed Annular Coated Inclusion (ACI) micromechanical method, a twodimensional finite element method (FEM) analysis of a three phase elastic material was adopted [15]. The unit cell used to determine the transverse Young's modulus of the composite is shown in Figure 2, where $W$ and $L$ denote the width and height of the unit cell, respectively. Plane strain conditions for the unit cell are assumed. The transverse Young's modulus was chosen for study as it is a matrix-dominated property that will best highlight the utility of the proposed micromechanical model. (The longitudinal results from the ACI model and standard MT model are almost identical as the predictions are dominated by the properties of the fiber when the fiber is the stiffest of the three phases.)

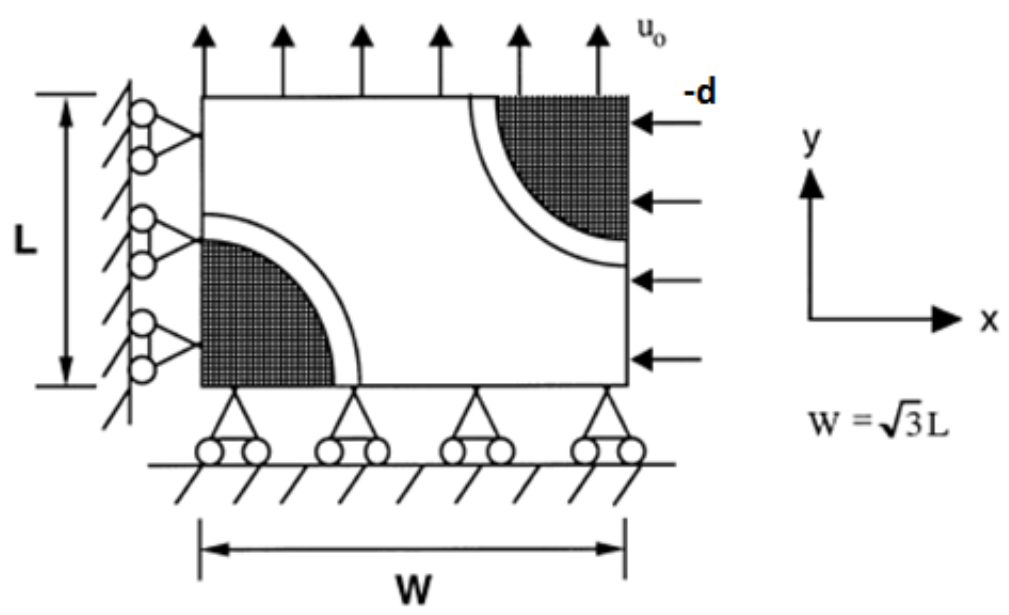

Figure 2. Unit cell analysis to determine the transverse Young's modulus [15]. 
Roller support boundary conditions are applied to the left-hand side and the bottom of the unit cell, respectively, as shown in Figure 2. The top face is given a prescribed uniform displacement of $u_{0}$. Further, to keep the right hand side remaining straight and parallel during deformation, a prescribed displacement of $-d$ at $x=W$ is applied. To determine the magnitude of the prescribed displacement $d$, the following traction boundary condition at $x=W$ should be approximately satisfied

$$
\sum T_{x}=0 \text { at } x=W
$$

where $T_{x}$ is the resultant nodal tractions on the right hand side of the unit cell. Practically, for a given uniform displacement of the top face of the cell $u_{0}$, one can determine the linear relationship between $\Sigma T_{x}$ and $-d$ such that an appropriate value of $d$ can be chosen, for a given set of material properties and geometries, such that the traction condition in (50) is approximately satisfied. Then the normal strain across the top face of the unit cell can then be written as

$$
\bar{\varepsilon}_{y}=\frac{u_{0}}{L}
$$

and the average resulting normal stress $\bar{\sigma}_{\text {y,ave }}$ on the top face of the unit cell can be determined based on the summation of the resultant nodal forces at $y=L$. From this, the transverse Young's modulus of the composite can be obtained as

$$
E_{T}=\frac{\bar{\sigma}_{y, a v e}}{\bar{\varepsilon}_{y}}
$$

\section{Results and Discussion}

For simplicity, unless otherwise specified we assume that the modulus values for the inclusion and matrix are 100 and 1, respectively, while depending on the particular 
arrangement being considered, the interphase modulus is 10 for the stiff interphase case and 0.1 for soft interphase case. In all cases we assume isotropic constituent materials with a Poisson ration of 0.3 .

The predictions for the transverse Young's modulus $E_{T}$ as a function of the volume fraction of the inclusion using the proposed Annular Coated Inclusion (ACI) model, the original multiphase Mori-Tanaka (MT) method, and the Finite Element Method (FEM) are shown in Figure 3, where the volume fraction of interphase is $10 \%$ for both cases. In the case where the interphase is stiffer than polymer matrix, the predictions of the ACI and the MT methods show excellent agreement and closely match FEM results. However, Figure $3 \mathrm{~b}$ shows significant deviation between the MT and ACI predictions for the case of the softer interphase (when the interphase modulus is equal to 0.1). In addition, the predictions of the ACI model more closely match the numerical results provided by FEM, while the predictions of the original MT method clearly overestimate the effective transverse modulus of the composite. This over-estimation when using the MT method is attributed to a "stress shielding" phenomenon, where the reinforcement provided by the inclusion is considerably reduced by the surrounding soft annular interphase; an effect which is captured in the proposed ACI method. Not surprisingly, a similar effect was noted in the companion paper for coated spherical inclusions [12]. 

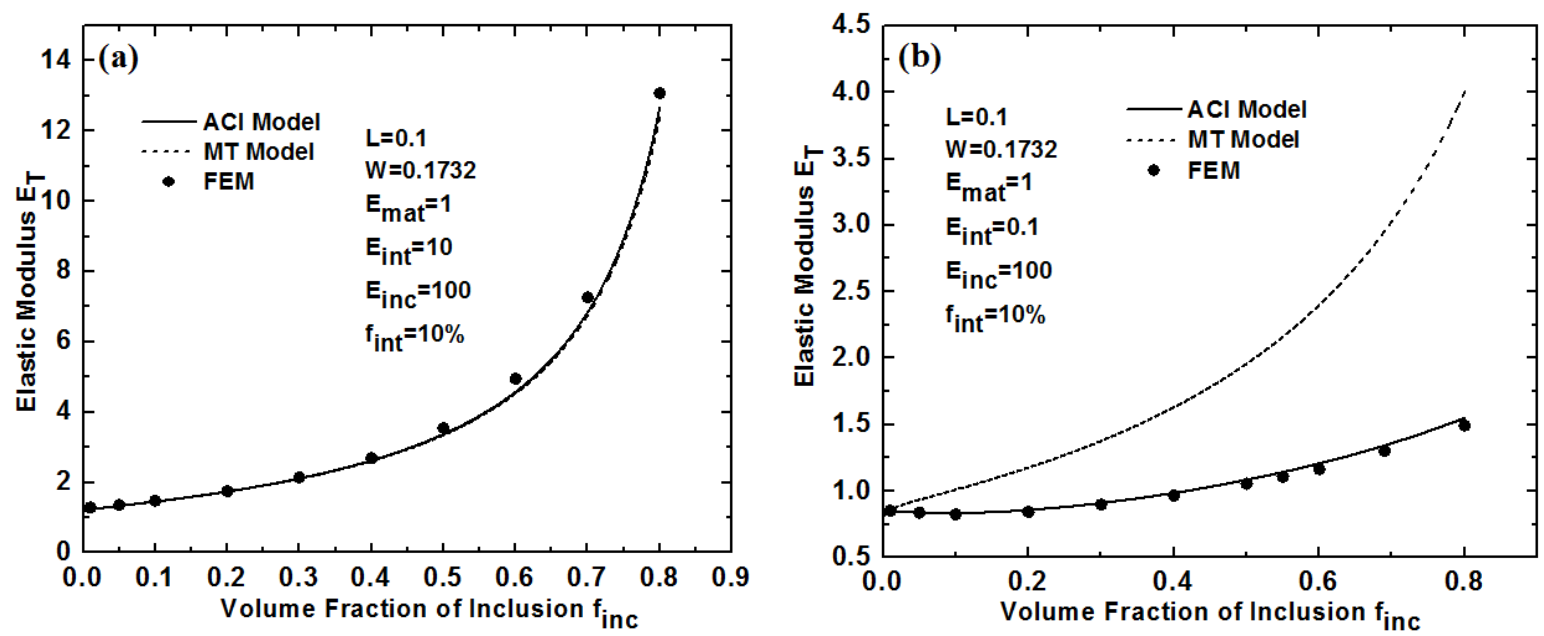

Figure 3. Predictions of transverse Young's modulus $\mathrm{E}_{\mathrm{T}}$ as a function of volume fraction of inclusion (a) interphase is stiffer than polymer matrix (stiff interphase case); (b) interphase is softer than polymer matrix (soft interphase case).

The influence of the interphase volume fraction on the overall composite elastic modulus is shown in Figure 4. The volume fraction of inclusion is held constant for this analysis at $10 \%$. As shown in Figure 4a, when the interphase is stiffer than matrix material, the composite modulus increases as the interphase volume fraction increases. For the case of a stiff interphase, the predictions from ACI model again show excellent agreement with predictions from both the MT model and FEM results, with only slight differences observable at larger interphase volume fractions. On the other hand, for the case of the soft interphase shown in Figure 4b, the ACI predictions exhibit lower predictions than those from the multiphase MT model. However, the ACI predictions are again close to the FEM numerical results, indicating that the annular soft interphase significantly softens the composite in a manner which is not effectively captured in the multiphase MT method. Interestingly, the difference between the ACI and MT predictions slightly decreases at 
higher volume fractions of interphase. The consistency of the ACI predictions with the FEM results reinforces the importance of accurately accounting for the shape of the annular interphase region, which is accurately captured via the dilute strain concentration tensor in the proposed three-phase Annular Coated Inclusion model.
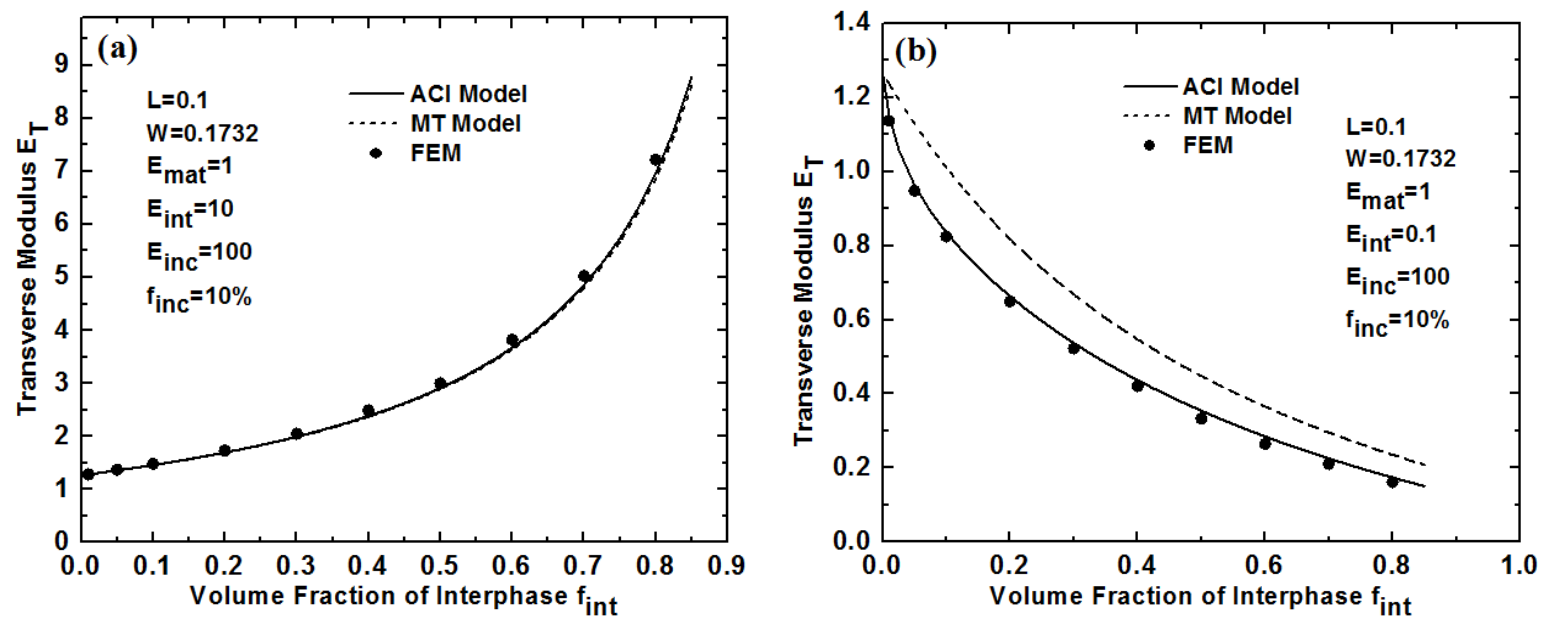

Figure 4. Predictions of transverse Young's modulus $E_{T}$ as a function of volume fraction of interphase (a) interphase is stiffer than polymer matrix (stiff interphase case); (b) interphase is softer than polymer matrix (soft interphase case).

Predictions for the transverse Young's modulus $E_{T}$ as a function of interphase modulus are shown in Figure 5. In Figure 5a, the volume fractions of inclusion and interphase are 5\% and $10 \%$, respectively, while in Figure $5 \mathrm{~b}$ the volume fractions of inclusion and interphase are $1 \%$ and $20 \%$. As before, the moduli of the inclusion and matrix are assumed to be 100 and 1, respectively. Both plots show that when the interphase modulus is smaller than 1 (softer than the polymer matrix), the predictions of the ACI method are lower than that of the multiphase MT method and agree well with FEM predictions. In addition, consistent 
with the results discussed above, the predictions of the two methods are quite close to each other (and the numerical results from the FEM model) when the interphase modulus is greater than 1 (stiffer than the polymer matrix). Note that the predictions of the two micromechanical approaches are identical where the interphase modulus is equal to 1 as this is equivalent to a two-phase composite (the interphase and matrix are identical). The appearance of a plateau when $E_{\text {int }}$ exceeds 100 in both cases indicates that the transverse behavior converges to a constant value for exceedingly large interphase moduli; a result that is consistent with the predictions obtained using FEM. Interestingly, Figure 5b shows that the differences between the MT and ACI models are smaller for the case of $f_{\text {inc }}=1 \%$ and $f_{\text {int }}=20 \%$, indicating that the discrepancies between model predictions are dependent on the constituent volume fractions and properties.
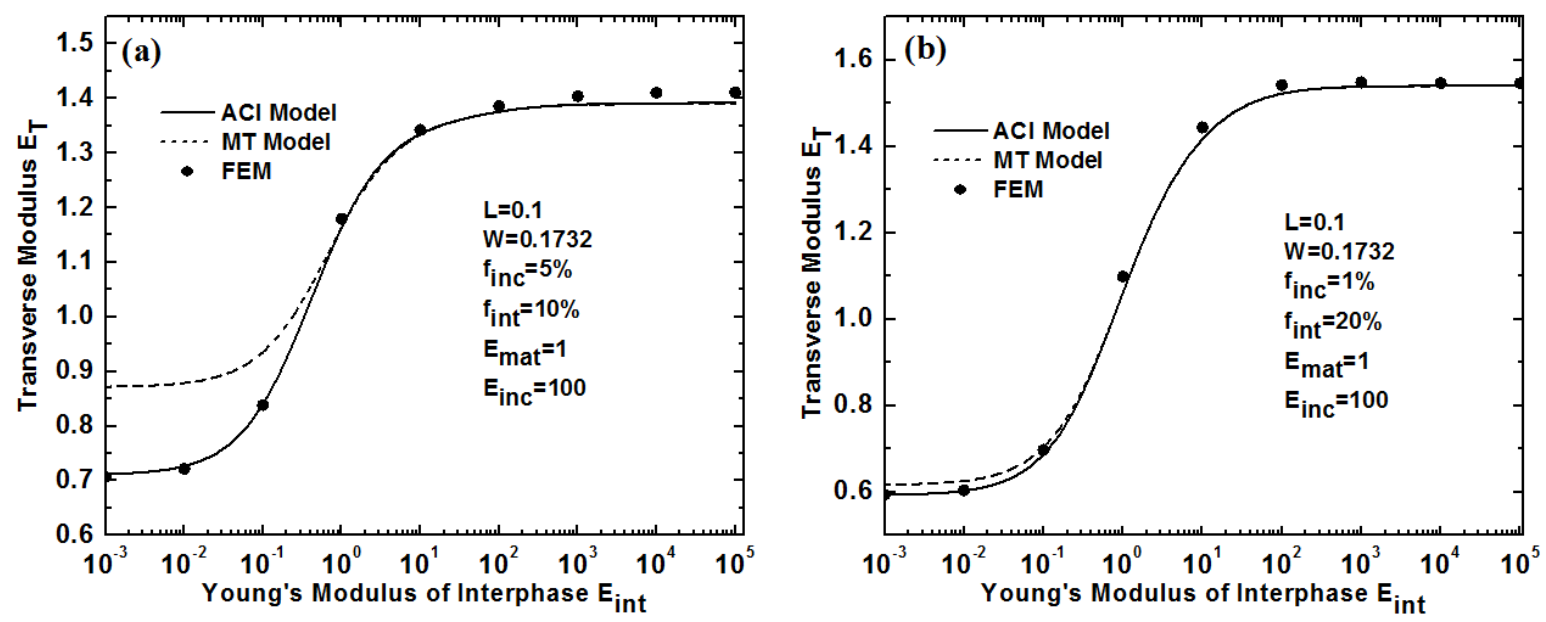

Figure 5. Predictions of transverse Young's modulus as a function of the modulus of the interphase (a) volume fractions of inclusion and interphase are 5\% and 10\%, respectively; (b) volume fractions of inclusion and interphase are $1 \%$ and $20 \%$, respectively. 
To further compare the influence of the interphase modulus on the effective composite properties predicted by the ACI model, two cases are examined in Figure 6: a matrix (modulus of 1) filled with either a stiff inclusion $\left(E_{\text {inc }}=1000\right)$ or a soft inclusion $\left(E_{\text {inc }}=0.01\right)$ and an interphase region or varying stiffness. Here the volume fractions of inclusion and interphase are $30 \%$ and 10\%, respectively. For the stiff inclusion case, the predictions are similar as before in that ACI results are lower than the MT model when the interphase is softer than the matrix, whereas the results from the two approaches starts to converge for interphase modulus greater than one. On the other hand, when the inclusion is soft $\left(E_{\text {inc }}=0.01\right)$, the $\mathrm{ACI}$ and MT predictions are quite close to each other until the modulus of the interphase is greater than that of the matrix, after which the ACI results are much higher than those from the multiphase MT model.

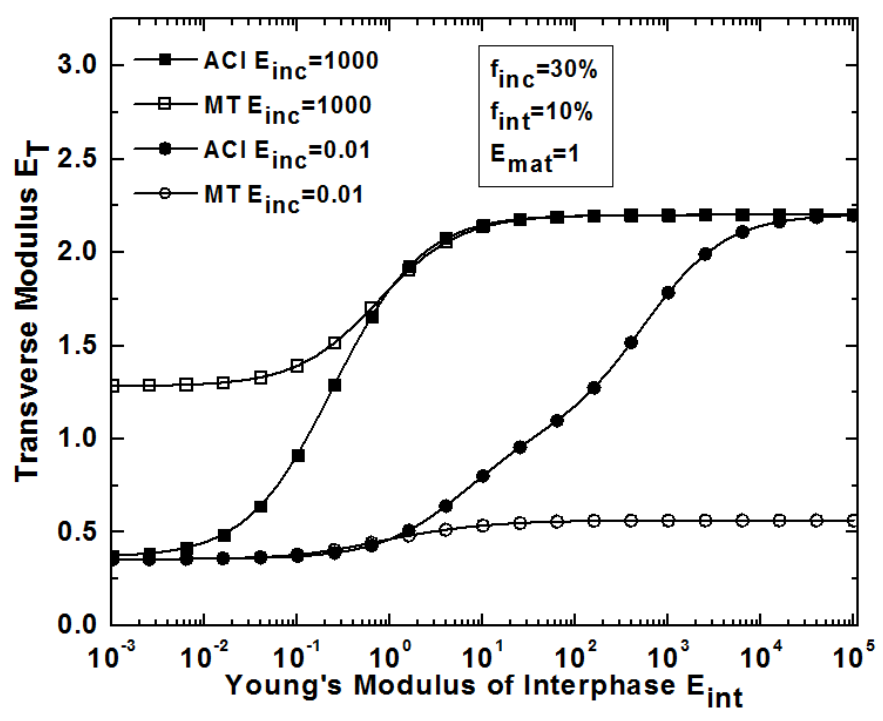

Figure 6. Predictions of transverse Young's modulus as a function of the modulus of the interphase with stiff inclusion $\left(E_{i n c}=1000\right)$ and soft inclusion $\left(E_{\text {inc }}=0.01\right)$. 
These results suggest that the impact of a soft inclusion is strongly screened by a stiffer annular interphase region, such that the predicted modulus by the ACI method is greater than the MT estimation where the interphase is treated as a separate phase. It also clear in Figure 6 that the ACI predictions for the composite modulus converge at the extreme minimum and maximum values of the interphase modulus, respectively, while the MT predictions approach separate plateaus which are a function of the inclusion modulus. This is again consistent with the "stress shielding" effect resulting from the annular interphase being captured in the ACI model.

For further comparison, the effective composite modulus prediction from each of the two micromechanical models, assuming a 3D random orientation of coated inclusions, is shown in Figure 7. Here the results show a trend similar to that of the transverse modulus (see Figure 6), where the standard multiphase MT model over-predicts the more accurate ACI model when the inclusion modulus is very small, while the MT model under-predicts the ACI model when the inclusion modulus is large. Both behaviors can be attributed to a "stress shielding" effect captured in the proposed ACI model. Note that the magnitude of the differences between the two models as shown in Figure 7 is somewhat masked due to the log-log nature of the plot. One difference between the behavior predicted for the transverse modulus shown in Figure 6 and the effective modulus predictions for the 3D randomly oriented coated inclusions shown in Figure 7 is that, as expected for the latter, the effective composite modulus predictions are dominated by the stiffest constituent material (as opposed to the transverse modulus being dominated by the matrix modulus). 


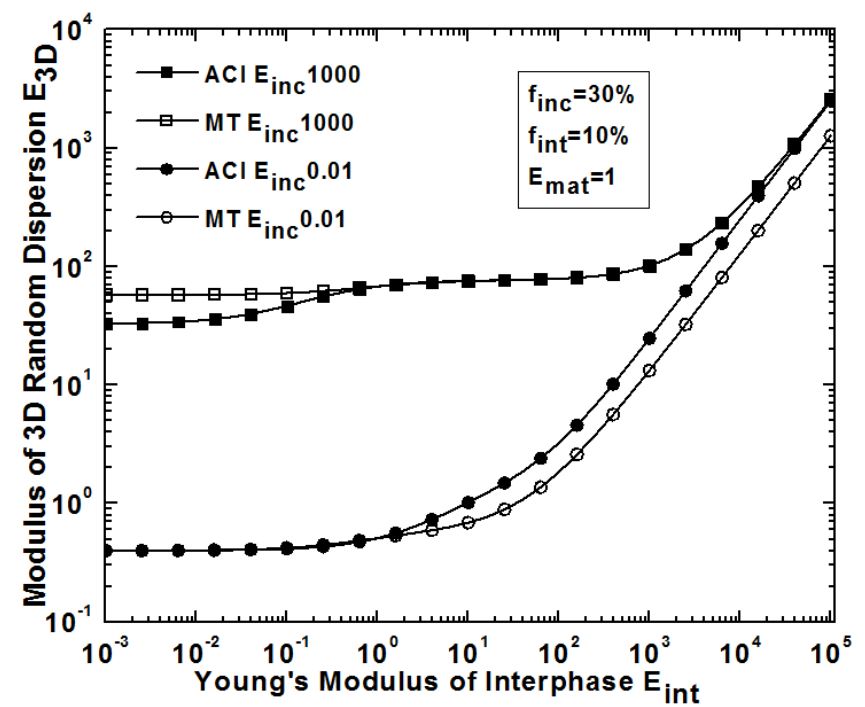

Figure 7. Micromechanical predictions of effective composite modulus with a 3D random orientation of coated inclusions as a function of the modulus of the interphase with stiff $\left(E_{i n c}=1000\right)$ or soft $\left(E_{i n c}=0.01\right)$ inclusions.

Lastly, the proposed ACI model can be used to interpret experimental composite data provided in the literature. As an example, Figure 8 shows a comparison of ACI modulus predictions for a polyimide PI/CNT nanocomposite with experimental results provided by Misiego et al. [26], where mechanical properties of PI/CNT films were measured using a Q800 TA Instruments Dynamic Mechanical Analyzer (DMA). Here the model assumes a three-dimensional random orientation of CNTs as there was no specific steps to align the CNTs during the sample fabrication described in the experimental work. From the experiment it is known that the polyimide modulus is $3.6 \mathrm{GPa}$ and the volume fraction of inclusion was variable up to $10 \%$. Starting with the known experimental data provided, composite modulus predictions (assuming a 3D random orientation of inclusions) can be 
made using the ACI model for different values of inclusion modulus, interphase modulus, and interphase thickness in an iterative process as highlighted in Figure 8.

As shown in Figure 8a, the trend in the experimental results as a function of volume fraction indicates that an effective CNT modulus of approximately $600 \mathrm{GPa}$ is necessary to match the experimental data (at least for assumed interphase thickness ratios of $t / r_{f}=2$ and an interphase modulus of $0.1 \mathrm{GPa}$ shown in Figure 8a). While larger assumed values for the interphase modulus and smaller interphase thickness ratios would result in lower estimates for the CNT modulus, the shape of the resulting predictions would be more linear than that shown in Figure 8a and do not seem to capture the experimental trends in the modulus data. Likewise, the shape of the experimental curve appears to show that a very low effective interphase modulus $(0.1 \mathrm{GPa})$ is necessary to capture the general behavior of the experimental data, with changes to the assumed values of the CNT modulus of $600 \mathrm{GPa}$ and the interphase thickness ratios of $t / r_{f}=2$ not able to capture the experimental trends. Similar trends are found for the interphase modulus (Figure 8b), where a value of $E_{\text {int }}=0.1$ GPa seems to best describe the observed behavior, and the interphase thickness ratio (Figure $8 \mathrm{c}$ ), with a value of $t / r_{f}=2$ best representing the character of the experimental data.

Thus comparing the ACI modulus predictions with the experimental data suggests that the trend in the data is well captured for a system where $E_{\text {inc }}=600 \mathrm{GPa}, E_{\text {int }}=0.1 \mathrm{GPa}$, and a ratio of interphase thickness to inclusion radius $t / r_{f}=2$. While this specific set of parameters is clearly not unique, and there was no attempt to optimize the iterative process highlighted here to select representative values to capture the trends in the experimental data, such an analysis illustrates the utility of applying an accurate micromechanical model for the interpretation of experimental data. Based on the results presented above and in the 
companion paper for coated spherical inclusions [12], the ACI approach derived here may be particularly useful for cases where the interphase region has a modulus lower than that of the matrix phase.
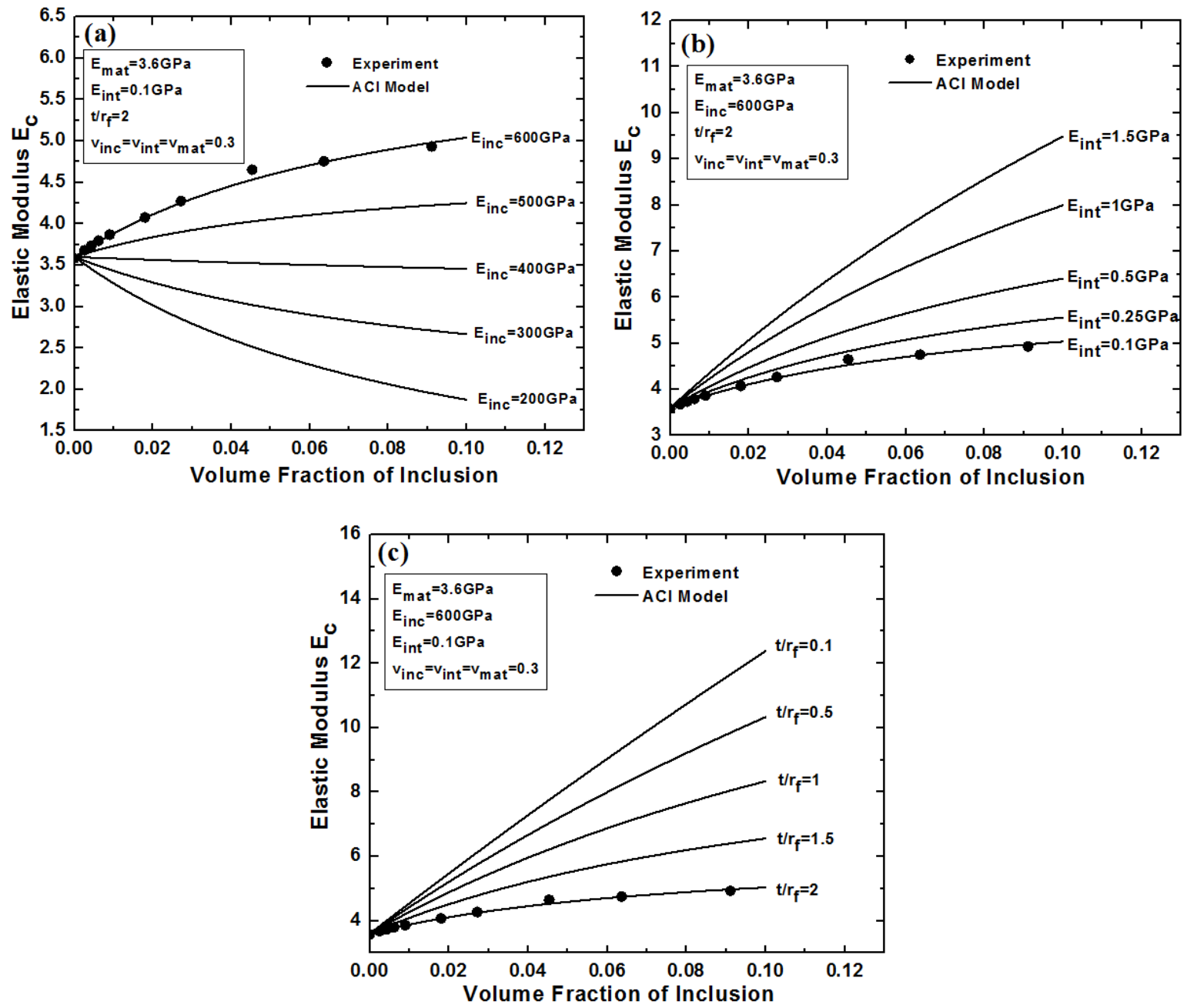

Figure 8. Comparison of ACI predictions with experimental results for Polyimide (PI)/CNT nanocomposite modulus [26] as a function of inclusion content for different (a) inclusion modulus; (b) interphase modulus; (c) ratio of interphase thickness to inclusion radius. 


\section{Conclusions}

Here we have derived an analytical solution for the calculation of the components of the dilute strain concentration tensor for a coated cylindrical inclusion following the procedure of Benveniste [14]. Appropriate auxiliary problems yield analytical solutions for the displacement, strain, and stress fields for a single coated cylindrical fiber. Based on these solutions, volume averaging is used to explicitly link the ratio of the average strain in each phase to the applied far-field strain, with the results then used to determine then individual elements of the dilute strain concentration tensor. This tensor can then be directly substituted into widely used micromechanical models such as Mori-Tanaka method to estimate the effective mechanical properties of the three-phase composite system with coated cylindrical inclusions.

A two-dimensional FEM analysis was used to benchmark the predictions of the transverse Young's modulus for a unidirectional composite obtained with the proposed Annular Coated Inclusion (ACI) method and the original multiphase Mori-Tanaka (MT) method. The analysis shows that the predictions of the two micromechanical methods are quite close when the interphase is stiffer than polymer matrix, with both techniques in good agreement with the FEM results. However, when the interphase is softer than the matrix, the Young's modulus predictions determined from the proposed ACI method closely matches the FE results, but are much lower than that of MT method. This finding is attributed to a "stress shielding" effect, as the original Mori-Tanaka (MT) method is not able to accurately capture the physicality of the annular interphase region surrounding the embedded inclusions. 
A benefit of the proposed technique is that the direct calculation of the components of the dilute strain concentration tensor allows for the technique to be readily applied to composites where the inclusions are prescribed an orientational distribution within the matrix, as highlighted by interpreting representative experimental data from the literature. An additional advantage of the approach is that it can be extended in a straightforward manner to enable a wide range of composite studies; for example, the incorporation of spring-layer interfacial conditions proposed by Hashin [20] to study the impact of imperfect interface bonding. Such models which specifically account for the geometry of this interphase region hold particularly application for polymer nanocomposites, and may provide insight into the impact of various approaches that seek to tailor the interphase region within these systems. 


\section{Acknowledgements}

Financial support for this work from the National Science Foundation (Grant No. 0846937) is gratefully acknowledged.

\section{References}

[1] Gibson RF. A review of recent research on mechanics of multifunctional composite materials and structures. Composite Structures. 2010;92(12):2793-2810.

[2] Du ML, Guo BC, Jia DM. Newly emerging applications of halloysite nanotubes: A review. Polymer International. 2010;59(5):574-582.

[3] Li B, Zhong WH. Review on polymer/graphite nanoplatelet nanocomposites. Journal of Materials Science. 2011;46(17):5595-5614.

[4] Wan C, Chen B. Reinforcement and interphase of polymer/graphene oxide nanocomposites. Journal of Materials Chemistry. 2012;22(8):3637-3646.

[5] Cheng X, Putz KW, Wood CD, Brinson LC. Characterization of local elastic modulus in confined polymer films via AFM indentation. Macromolecular Rapid Communications. 2015;36(4):391-397.

[6] Barber AH, Cohen SR, Eitan A, Schadler LS, Wagner HD. Fracture transitions at a carbonnanotube/polymer interface. Advanced Materials. 2006;18(1):83-87.

[7] Lin Y, Ehlert G, Sodano HA. Increased interface strength in carbon fiber composites through a ZnO nanowire interphase. Advanced Functional Materials. 2009;19(16):2654-2660.

[8] Jeziórska R, Zielecka M, Gutarowska B, Żakowska Z. High-density polyethylene composites filled with nanosilica containing immobilized nanosilver or nanocopper: Thermal, mechanical, and bactericidal properties and morphology and interphase characterization. International Journal of Polymer Science. 2014;2014:1-13. 
[9] Mori T, Tanaka K. Average stress in matrix and average elastic energy of materials with misfitting inclusions. Acta Metallurgica. 1973;21(5):571-574.

[10] Weng GJ. The theoretical connection between Mori Tanaka theory and the Hashin Shtrikman Walpole bounds. International Journal of Engineering Science. 1990;28(11):1111-1120.

[11] Hori M, Nemat-Nasser S. Double-inclusion model and overall moduli of multi-phase composites. Mechanics of Materials. 1993;14(3):189-206.

[12] Wang Z, Fisher FT. Annular Coated Inclusion model and applications for polymer nanocomposites - Part I: Spherical inclusions. Mechanics of Materials. 2016;Under review. [13] Luo HA, Weng GJ. On Eshelby's S-tensor in a three-phase cylindrically concentric solid, and the elastic moduli of fiber-reinforced composites. Mechanics of Materials. 1989;8(2-3):77-88. [14] Benveniste Y, Dvorak GJ, Chen T. Stress fields in composites with coated inclusions. Mechanics of Materials. 1989;7(4):305-317.

[15] Fisher FT, Brinson LC. Viscoelastic interphases in polymer-matrix composites: Theoretical models and finite-element analysis. Composites Science and Technology. 2001;61(5):731-748. [16] Bradshaw RD, Fisher FT, Brinson LC. Fiber waviness in nanotube-reinforced polymer composites-II: Modeling via numerical approximation of the dilute strain concentration tensor. Composites Science and Technology. 2003;63(11):1705-1722.

[17] Cherkaoui M, Sabar H, Berveiller M. Elastic composites with coated reinforcements: a micromechanical approach for nonhomothetic topology. International Journal of Engineering Science. 1995;33(6):829-843.

[18] Seidel GD, Lagoudas DC. Micromechanical analysis of the effective elastic properties of carbon nanotube reinforced composites. Mechanics of Materials. 2006;38(8):884-907.

[19] Jiang Y, Yang H, Tohgo K. Three-phase incremental damage theory of particulate-reinforced composites with a brittle interphase. Composite Structures. 2011;93(3):1136-1142. 
[20] Hashin Z. Thermoelastic properties of particulate composites with imperfect interface. Journal of the Mechanics and Physics of Solids. 1991;39(6):745-762.

[21] Fisher FT, Bradshaw RD, Brinson LC. Fiber waviness in nanotube-reinforced polymer composites-I: Modulus predictions using effective nanotube properties. Composites Science and Technology. 2003;63(11):1689-1703.

[22] Christensen RM, Lo KH. Solutions for effective shear properties in three phase sphere and cylinder models. Journal of the Mechanics and Physics of Solids. 1979;27(4):315-330.

[23] Savin GN. Stress Concentration around Holes. New York: Pergamon Press Inc.; 1961.

[24] Walpole LJ. On the overall elastic moduli of composite materials. Journal of the Mechanics and Physics of Solids. 1969;17(4):235-251.

[25] Bolcu D, Rizescu S, Ursache M, Bîzdoacă GN, Stănescu MM, Rinderu P. Spectral decomposition of the elasticity matrix. UPB Scientific Bulletin, Series A: Applied Mathematics and Physics. 2010;72(4):217-232.

[26] Misiego CR, Pipes RB. Dispersion and its relation to carbon nanotube concentration in polyimide nanocomposites. Composites Science and Technology. 2013;85:43-49. 


\section{Appendix}

As discussed in Section 2.3, for the solution of the transverse shear loading auxiliary, the unknown displacement field constants in (25) can be determined by solving the system of equations which can be written in the matrix form such that $[A] \cdot[x]=[y]$, where $[x]=\left[A_{2}^{f}, A_{2}^{g}, C_{2}^{m}, B_{2}^{g}, C_{2}^{g}, B_{2}^{m}, D_{2}^{f}, D_{2}^{g}\right]^{T},[y]=\left[0,0,2 \mu_{x y}^{g},-2 \mu_{x y}^{g}, 0,0,0,4 \mu_{x y}^{g} \mu_{x y}^{m},-2\right]^{T}$, and the matrix $[A]$ can be written as

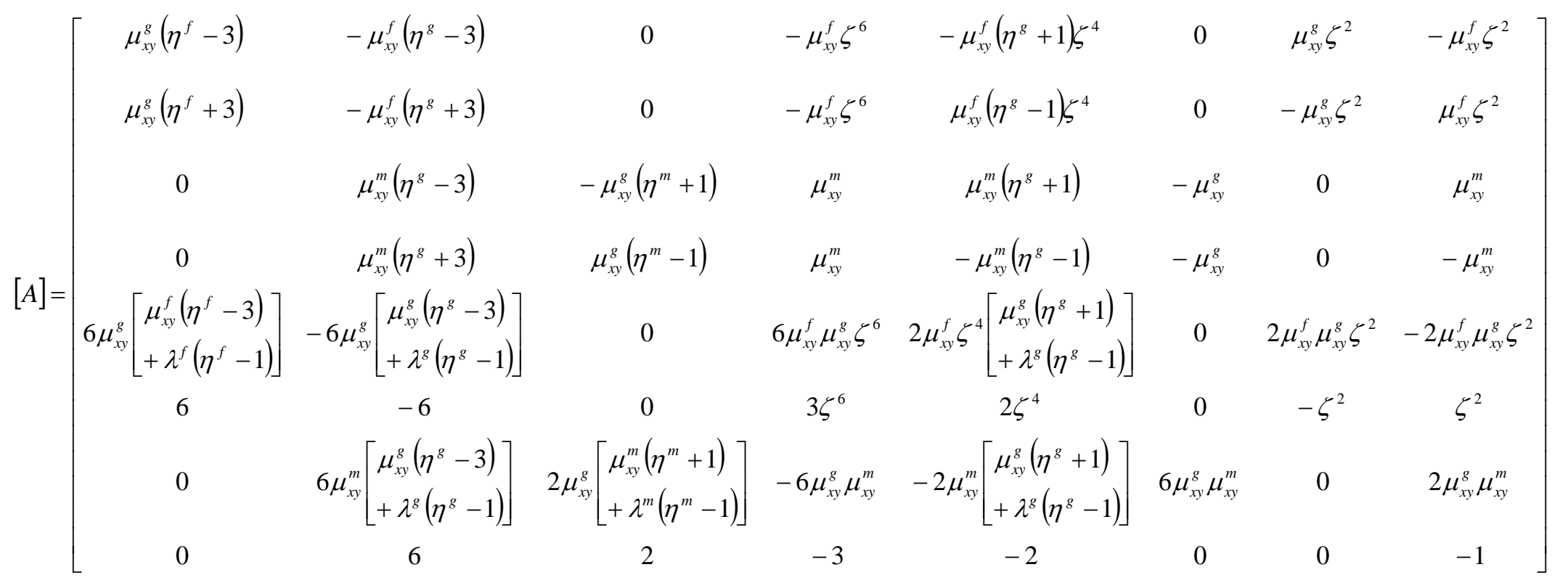

where $\zeta=b / a$. 

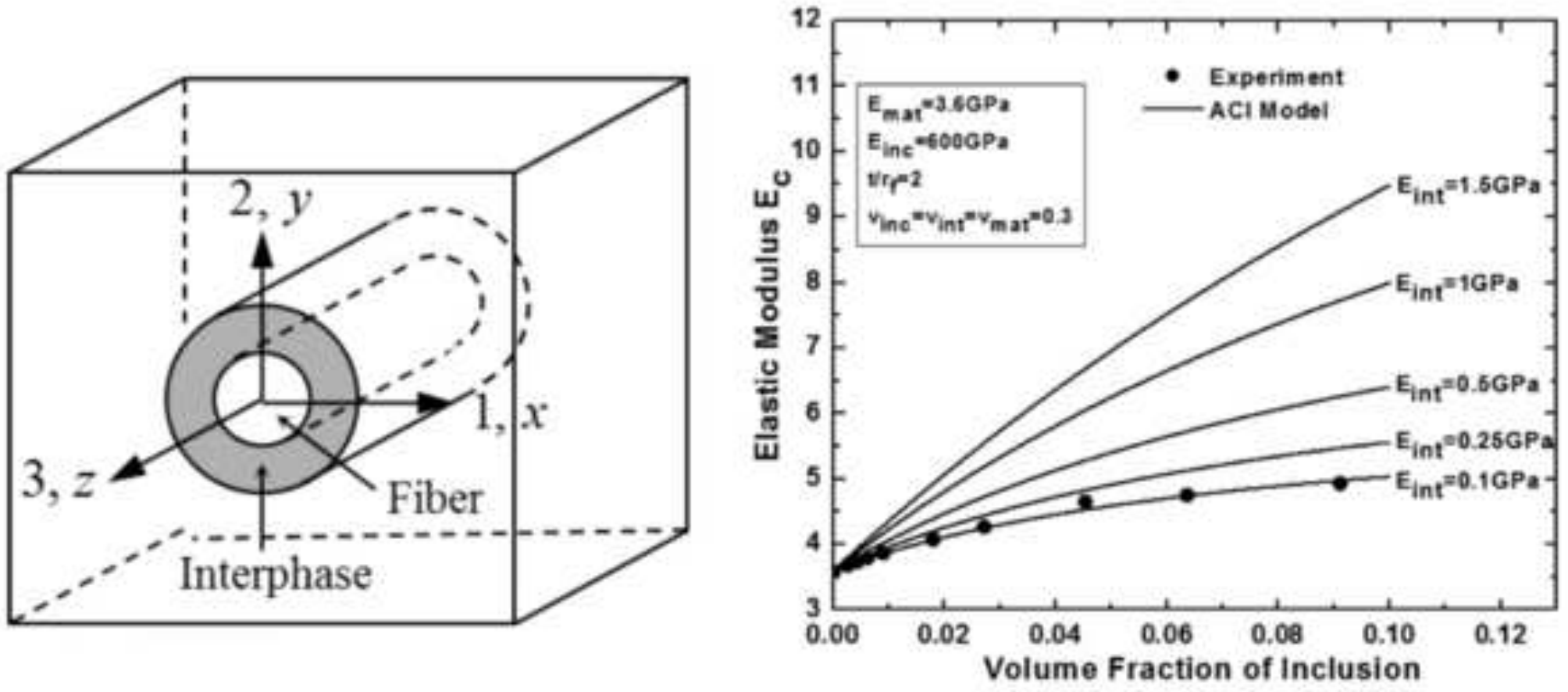